\title{
Review
}

\section{Tremor in Parkinson's Disease May Arise from Interactions of Central Rhythms with Spinal Reflex Loop Oscillations}

\author{
Dimitri Anastasopoulos $\mathrm{a}, \mathrm{b}, *$ \\ ${ }^{a}$ Department of Neurology, University of Ioannina, Ioannina, Greece \\ ${ }^{\mathrm{b}}$ Akutnahe Rehabilitation, Kantonsspital Baden, Baden/Bad Zurzach, Switzerland
}

Accepted 4 December 2019

\begin{abstract}
It is commonly believed that tremor, one of the cardinal signs of Parkinson's disease, is associated with cerebellothalamo-cortical oscillations set off by the dopamine-depleted basal ganglia networks. The triggering mechanism has been, however, not entirely delineated. Several reports have pointed to the relevance of interactions with peripheral/spinal mechanisms to tremor generation. Investigations of motor unit synchronization and discharge patterns suggested that exaggerated beta-band oscillations may intermittently reach alpha-motoneurons and modulate low-amplitude membrane oscillations due to spinal loop transmission delays. As a result, the spinal reflex loop will oscillate more vigorously and at a lower frequency and, in turn, entrain larger transcortical loops. Motoneurons may thus represent the specific generator "node" in a tremor network encompassing both cerebral and peripheral/spinal recurrent circuits.
\end{abstract}

Keywords: Parkinsonian tremor, motor unit synchrony, spike doublets, beta oscillations

\section{INTRODUCTION}

The exact origins and pathophysiology of parkinsonian tremor, though intensively investigated, are still disputed. The widely accepted model of dysfunctional basal ganglia networks cannot adequately explain this prominent motor sign [1]. Tremor is characterized by involuntary, rhythmic and alternating movement of a body part, mostly of a limb, at 4-8 Hz. It may occur at rest, when maintaining a posture against gravity or during voluntary muscle contraction. Tremor is not always present in patients and its severity does not correlate with other motor or non-motor symptoms of the disease [2]. Patients

\footnotetext{
${ }^{*}$ Correspondence to: Dimitri Anastasopoulos, Department of Neurology, University of Ioannina, 45110 Ioannina, Greece. Tel.: +30 6944707069/+41 798599463; Fax: +41 564863039; E-mail: danastas@nurs.uoa.gr.
}

are accordingly classified as belonging to the tremordominant or the akinetic-rigid subtype [3]. Also, it does not progress at the same rate as the other symptoms of Parkinson's disease (PD), correlates poorly with the extent of dopamine depletion in the basal ganglia and responds less well to dopaminergic treatment [4]. These observations suggest that tremor has a different pathophysiology from that of bradykinesia and rigidity [5]. Though most investigators agree that PD tremor is generated by one or several supraspinal oscillators, there are several reports suggesting that spinal/ peripheral mechanisms interact with central rhythms and may participate in its production. Several new aspects of such interactions between common synaptic inputs onto populations of motoneurons have emerged by analyzing motor unit synchronization and discharge patterns from tremulous limb muscles in patients. 


\section{CEREBRAL OSCILLATIONS AND PARKINSONIAN TREMOR}

Many investigations have tried to identify the oscillatory cerebral circuit implicated in parkinsonian tremor generation by relating cycle-to-cycle tremor rhythms to discharges of single neurons or the activity of large neuronal populations in patients or animals rendered parkinsonian. Some thalamic nuclei (ventralis intermedius and ventralis oralis posterior) are considered to be involved in a loop causing the tremor or even represent the tremor pacemaker because cells showing a large amount of correlated activity at tremor frequency have been documented in this region [6] and tremor is substantially reduced by a small lesion or deep brain stimulation (DBS) in this area [7-10]. Rhythmic neural activity at a frequency in the range of parkinsonian tremor (tremor-related activity) has been also reported in the internal segment of globus pallidus (GPi) [11], the main basal ganglia output structure. The significance of this finding was however later disputed, as signals simply oscillating within a narrow frequency range may be completely uncorrelated. Indeed, when the temporal coupling of neuronal and tremulous muscular activity was estimated by computing the coherence spectrum between Gpi single units and surface EMG (sEMG) pairs, synchronized oscillations were only transient [12]. It is therefore generally believed that pallidal neurons cannot be viewed as the tremor generators [13]. All the same, focal lesions at the GPi produce significant improvements in tremor [14]. The paradox has been tackled by recent work showing that tremor amplitude-related functional MRI signal increases are localized in cerebellum, thalamus and motor cortex while those localized in basal ganglia are time-locked to the onset of high-amplitude tremor episodes [15]. Accordingly, oscillations across the cerebello-thalamo-cortical circuit are thought to be entrained or triggered by altered basal ganglia output and these interactions are supposed to occur at the level of motor cortex (the "dimmer-switch" model) [16]. Notably, rhythmic activities centred around $20 \mathrm{~Hz}$ (beta-band oscillations) are ubiquitous and abnormally synchronized throughout the corticalbasal ganglia circuitry in PD [17-19]. Indeed, when these activities are suppressed by high-frequency DBS or dopamine replacement therapy, parkinsonian symptoms but not tremor, are ameliorated in patients and MPTP-treated primates [20]. It is therefore unclear whether and how pathological beta activity would drive the cerebello-thalamo-cortical circuit into tremor. It has been further argued that the "dimmer-switch" model is incomplete as neuronal activity correlated to tremor has been found in nucleus subthalamicus [21-23] and subthalamic stimulation at near-to tremor frequency entrained resting tremor in patients. Still, correlated activity may represent responses to afferent proprioceptive feedback [24]. The extent of sensory contributions to the central tremor-frequency oscillation is thus under debate. Also, the measured increases or decreases in beta or tremor activity in nucleus subthalamicus may have not been related to tremor onset but mainly to tremor maintenance and, therefore, the true temporal relationship between oscillatory activity in basal ganglia output structures and tremor may have been missed [25]. Also, findings of phase entrainment need not necessarily imply that the modulatory stimulus is applied to the generator itself. For example, phase entrainment of tremor by imposed flexion extension movements of patients' tremulous limbs (see below) does not undoubtedly prove the reflexive origin of parkinsonian tremor [26].

\section{AFFERENT INPUTS IN PARKINSONIAN AND PHYSIOLOGICAL TREMOR}

Based on transient changes on the tremor phase brought about by kinaesthetic or electrical stimulation, it has been claimed that activation of segmental reflex loops are important in the generation of various types of PD tremor. Short mechanical [27-29] or electrical $[30,31]$ perturbations changed the timing of the following tremor cycles and locked them to the stimulus ("resetting"). It has been generally assumed that only perturbations delivered to the pacemaker but not to 'secondary', downstream links or coupled paths will produce these effects. Similar behavior is exhibited by the cardiac atrial pacemaker when it is depolarized by external electrical shocks. However, transient resetting of the whole system of shorter or longer duration can be observed on applying perturbations, if strong enough, in different sites of tightly interconnected oscillators. For example, transcranial magnetic stimulation of motor cortex can equally reset parkinsonian tremor [32-34].

Alternating flexion-extension movements of just a few degrees at the wrist joint can entrain EMG activity connected with tremor in PD patients [26]. With movements close to the spontaneous frequency of tremor, muscle activity could be locked in many cases to the rate of the driving imposed movement, 
often over an appreciable range of frequencies, while its phase changed gradually from resisting to assisting the driving stimulus. These findings were interpreted by the authors as evidence that PD tremor is shaped by the interaction of afferent activity with central oscillations.

Afferent input from muscle spindles onto motoneurons is likewise associated with the generation of physiological tremor, i.e., the natural, low amplitude oscillations of the outstretched hands when normal subjects try to maintain a horizontal upper limb posture against gravity. Similar low amplitude oscillations at $6-12 \mathrm{~Hz}$ (a "ripple") is superimposed upon normal, isometric (constant strength) voluntary muscular contractions. Afferent input is thought to contribute to physiological tremor because each Ia fibre from a muscle spindle synapses on almost every single motoneuron in the homonymous motoneuronal pool and inhibits through interneurons motoneurons innervating antagonists. As a result of the common synaptic input, motor units can be synchronized and consequently generate tremor when, for example, the spinal stretch loop begin to oscillate [35]. Indeed, a brief downward, stepwise displacement to the outstretched finger gives rise to a train of approximately sinusoidal movements of it, in phase with bursts of motor unit discharges recorded by means of sEMG over its extensor muscle [36]. Also, direct recordings of normal human muscle spindle afferent activity showed that the segmental stretch reflex is extremely sensitive to the minute oscillations of physiological tremor [37]. The muscle spindles discharge only once during each tremor cycle and tend to synchronize the motor outflow producing rhythmical contractions in such a way as to reinforce the inflow of muscle spindle endings (positive feedback). The hypothesis of motor unit synchronization as the critical mechanism of physiological tremor generation has been questioned because conduction delays in operation of the monosynaptic spinal stretch reflex (around 20-25 ms) have been considered too short to generate a tremor in the frequency range of $7-11 \mathrm{~Hz}$. Still, the time delay of the reinforcing signal transmission loop consists of not only the afferent and efferent conduction delays but also of the time interval between the initiation of the muscle twitch and the grouped discharges of muscle spindles, occurring at the steepest decaying phase of the muscle twitch (Fig. 1A). This latter time interval is much longer than the former, i.e., approximately $100 \mathrm{~ms}$ in muscles of normal human hand [38], such as to allow for tremor-generating synchronization of motor neuron discharges at around $9 \mathrm{~Hz}$.
In conclusion, motor unit synchronization through proprioceptive input has been recognized as a mechanism contributing to physiological tremor. Similarly, afferent input interactions with central oscillators have been shown to exist and to be capable of entraining parkinsonian tremor. However, these investigations have not given a clear idea about the impact of the putative interactions upon the mechanism of tremor generation in PD.

\section{MOTOR UNIT FIRING SYNCHRONY AND PATTERNS IN PATIENTS}

Tremulous muscle contractions can be produced only when motor units discharge, particularly when they discharge rhythmically and synchronously in groups. The study of motor units innervating trembling muscles may thus yield information about tremor generating mechanisms. Generally, neurons are thought to fire together (they are "synchronized", Fig. 1) if they receive shared synaptic input at a time when chance fluctuations in membrane potential bring them near to threshold [39]. Thus, detection of synchrony within a neuronal population tells us that a subset of neurons may share synaptic input.

Synchrony of firing motor unit pairs in PD patients has been investigated in relatively few studies suggesting the involvement of inhibitory mechanisms (i.e., reduced descending inhibition) [40, 41]. Further, abnormally irregular firing patterns of motor units in PD have been described [40-43]. They consist of pairs of spikes ("doublets") with interspike intervals (ISI) between 30-60 ms (Fig. 2). These repetitive motoneuron discharges have been thought to be due to prolonged excitatory state and to represent a mechanism contributing to tremor amplitude. Their link, if any, to enhanced motoneuronal synchronization has remained however an open question.

In two recent studies, Christakos et al. [44] and Agapaki et al. [45] investigated motor unit synchrony and firing patterns during force (isometric contractions), postural and resting tremor in a large number of mildly affected patients with tremor dominant PD. The discharges of single motor unit spikes were recorded with needle electrodes inserted into the muscles involved in tremor production. Rhythmical force fluctuations (force tremor) exerted when executing weak quasi-isometric contractions against a force transducer and surface electromyogram (sEMG) from the same muscle were recorded simultaneously. Postural or resting tremor were investigated 

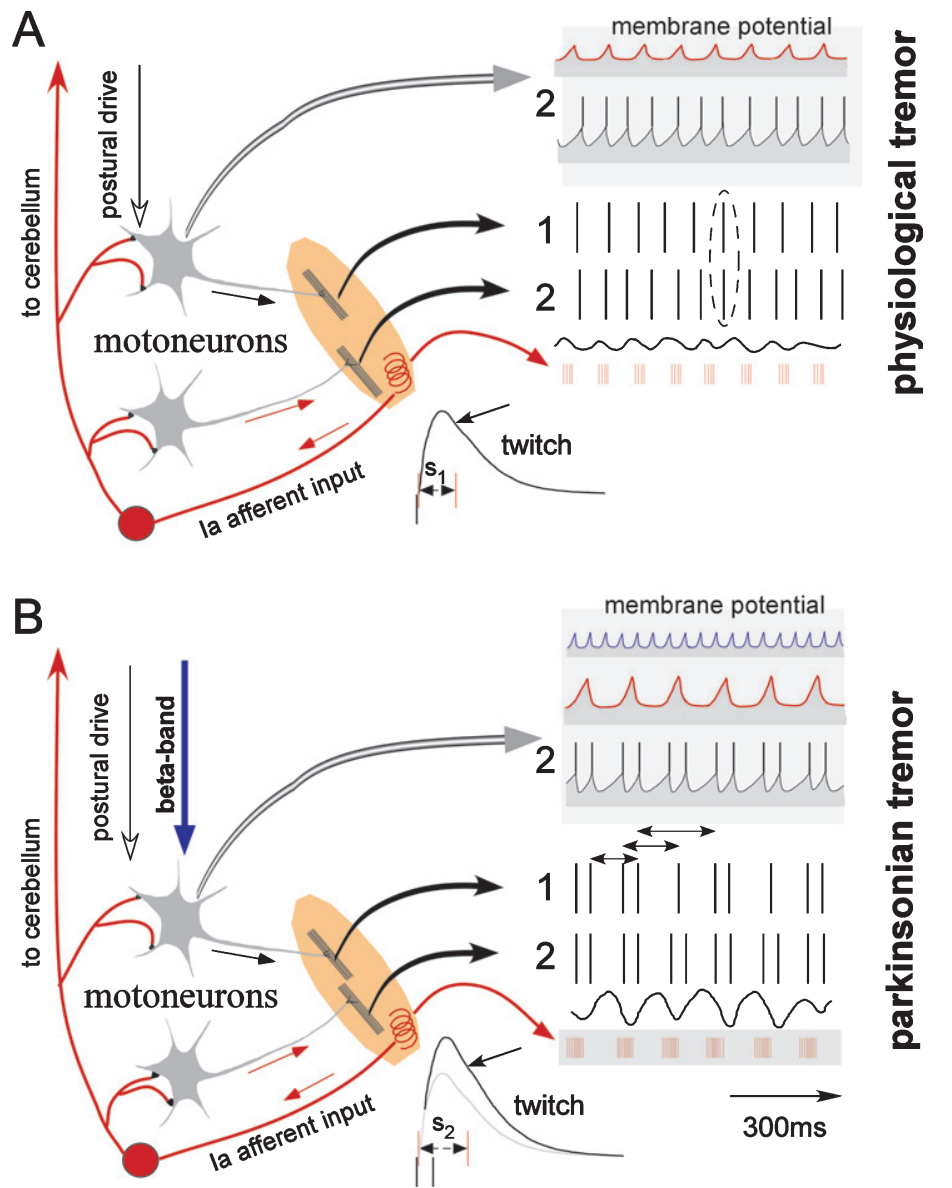

Fig. 1. Schematic diagram of the proposed dynamic interactions between descending beta-band oscillations and the spinal reflex loop in triggering parkinsonian force and postural tremors. Left panels depict the anatomical substrate. In A, motor units 1 and 2 discharge at 10 and 12 spikes/s respectively in response to descending postural drive. The minute tremulous limb/muscle oscillations (physiological tremor/normal-like tremor during epoch-I in patients) are sensed by muscle spindles (shown as a spiral, neural afferent activity depicted in red). Motor units discharge sometimes concurrently (vertical ellipse) as they receive common synaptic input from the spindles. The expected membrane potential fluctuations (motor unit 2 taken as example) due to the spindle input occur mainly during the stretch phases of tremor and are shown at the uppermost trace (within a gray background), while those at the axon hillock reaching firing threshold are shown below them. Delays in the spinal reflex loop (red arrows) include not only the conduction time but also the interval between the beginning of the synchronized motor unit contractions and the activation of muscle spindles $\left(S_{1}\right)$. The burst of the afferent neural discharges is generated around the steepest phase of the twitch relaxation curve (signaling the velocity of lengthening, oblique arrow). In this example a total delay of $125 \mathrm{~ms}$ has been assumed, resulting in tremor at approximately $8 \mathrm{~Hz}$. In B, excitatory postsynaptic potentials in response to descending exaggerated beta-band oscillations are thought to interact with those invoked by the spindle input. Motoneurons are expected to fire doublets, as threshold may be approached twice during each tremor cycle, i.e., when both inputs impinge on the motoneuronal membrane concurrently (temporal summation). In turn, due to the steeper relaxation curve of the superimposed "double" muscle force twitches (illustrated on top of the single twitch in the lowest part of the figure) the afferent Ia inflow (shown in lowest trace in gray background) and, in consequence, motor unit synchronization are expected to be enhanced. At the same time, the reflex loop delay will be extended by the interposed ISI within doublets (oblique arrow, $\mathrm{S}_{2}$ ) to approximately $160 \mathrm{~ms}$ and the frequency of tremor oscillations will be reduced to $6 \mathrm{~Hz}$. Because of the enhanced afferent spindle drive all spikes in excess of the tremor frequency are "bound up" in the formation of doublets/triplets, time-locked to tremor (motor unit 2, 6 doublets/s; motor unit 1, 4 doublets and 2 "singlets"/s). Ascending collaterals of the Ia afferents transmit these changes and entrain the cerebello-thalamo-cortical circuit, the oscillatory activity of which, in turn, maintains and amplifies the motoneuronal firing rhythm. Horizontal arrows along the spike train of motor unit 1 define second order interspike intervals.

by recording the acceleration of tremulous upper limbs when assuming a horizontal posture or when completely relaxing respectively, together with motor unit spikes and sEMG. Synchrony between motor units was evaluated by correlating a spike train from one single motor unit (decomposed manually from intramuscular EMG) to force/acceleration/sEMG signals in the frequency domain (called unit-to- 
aggregate coherence, Fig. 3). The delay ("phase") of synchronized motor units to tremor (tremorrelated and "phase-locked", i.e., showing coherence to tremor) was evaluated by cross-correlation computations between the spikes of the motor unit and force/acceleration/sEMG in the time domain (Fig. 3). Such cross-correlograms can be constructed when the spikes are used as a trigger for averaging the tremor signal. It had been earlier shown that the unit-to-aggregate coherence function can correctly capture the average strength of unit-to-unit synchrony (i.e., between correlated motor unit pairs) within a large sample of neuronal population activities [46]. Thus, instead of recording and correlating a large number of motor unit pairs, the average population synchrony could be estimated by calculating the coherence spectrum between only one motor unit and surface EMG/tremor, reducing in this way the recording time and discomfort in patients. By contrast, the aggregate-to-aggregate coherence function between subpopulations of motor units belonging to a muscle (for example utilizing so-called "composite" spike trains obtained from multichannel sEMG signals, see [47]) can detect correlations indeed non-invasively but probably at the expense of overestimating their strength and frequently introducing saturation effects. The discharge pattern irregularity of motor units in patients was quantified by means of high-order ISI analysis [48]. Denoting as a first-order interval the elapsed time from a spike to the next following spike, a second-order interval is defined as the elapsed time between a spike and the second following spike, etc. (Fig. 1). The times of occurrence of doublets or triplets as a fraction of the number of tremor cycles in the spike train ("incidence") was estimated from the probability density of ISIs around the tremor period in second- and third-order histograms respectively. This relationship resulted from the observation that doublets and triplets were timelocked to overt parkinsonian tremor such that the sum of a short ISI within a doublet and the following longer ISI added up to one tremor period (Fig. 1). Similarly, the sum of two short ISIs within a triplet and the longer ISI following them added up to one tremor period (Fig. 3) [49].

In each of the two-minute recordings of force/ postural/ rest tremor in patients, intervals of overt tremor (epoch-II) were randomly interspersed with intervals of low amplitude tremor (epoch-I) resembling physiological force and postural tremor in normal subjects. While the average firing rate of the motor unit under investigation remained fairly con-

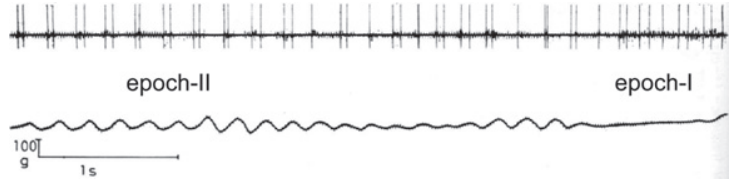

Fig. 2. Abnormal motor unit firing pattern in a patient. A motor unit from the first dorsal interosseous muscle was recorded when the patient was trying to exert a constant force with his finger against a strain gauge. Rhythmical doublets or doublets interchanged with single discharges characterize the interval of overt tremor (epochII). Note that the firing pattern is more regular and the rate (around 10-12 spikes/s) does not change appreciably when the tremor subsides (epoch-I; from [43]; Fig. 4, with kind permission).

stant between epochs, its discharge pattern changed dramatically (Fig. 2): during the normal-like epoch-I motor units fired more or less regularly at their intrinsic rate (commonly referred to as a stochastic process) and only occasionally were spikes in a fixed phase relationship to tremor (schematic representation in Fig. 1A). In contrast, during epoch-II, the units fired rhythmically doublets and/or triplets, time-locked to tremor, suggesting the impingement of strong modulatory synaptic inputs (schematic representation in Fig. 1B). Motor unit-to-tremor coherence at the overt tremor frequency (between 4.5 and $7.0 \mathrm{~Hz}$ ) was thereby significantly enhanced (at least 200\%) as compared to that during normal-like epoch-I and to coherence in normal subjects. Notably, the tremor frequency during normal-like epoch-I was approximately $2 \mathrm{~Hz}$ higher than during epoch-II, being close to the frequency of force and postural tremor in normal subjects (i.e., physiological tremor).

The mean short ISI duration within doublets/triplets during a two minutes recording varied between 40 and $50 \mathrm{~ms}$. Thus, doublets/triplets in patients should not be confused with repetitive discharges of normal animal and human motoneurons due to delayed depolarization because the latter have considerably shorter duration (between 2-20 ms) and occur mostly at the beginning of contractions [50, 51]. Notably, the mean short ISI between doublets was not different from that between triplets occurring during the same two-minute record or between doublets/triplets from any other record in the same patient, in posture or at rest (i.e., in recordings separated by several minutes up to half an hour). Further, the incidence of doublets and triplets during epoch-II (as a measure of the discharge pattern irregularity) was directly related to the intrinsic rate of the motor unit such that all spikes in excess of the tremor frequency participated in the formation of doublets/triplets. For example, when the motor 

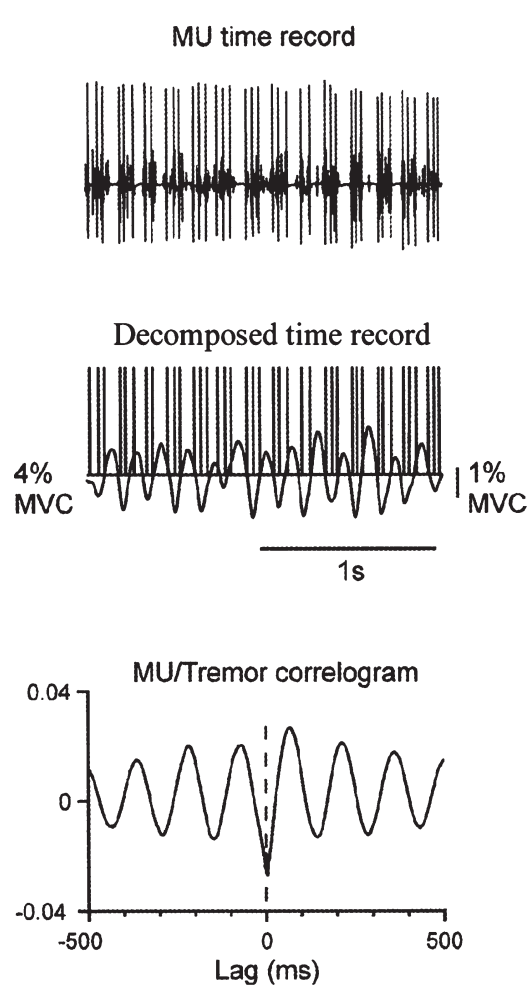

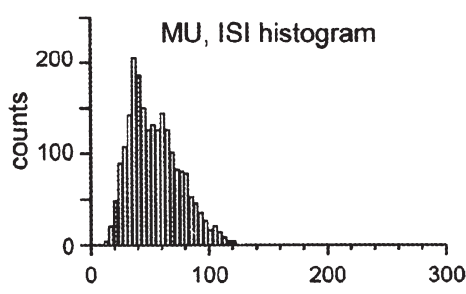

MU, 3rd-order interval histogram
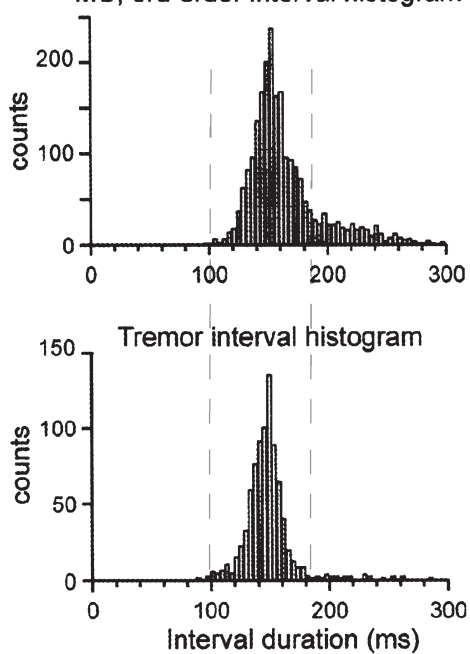
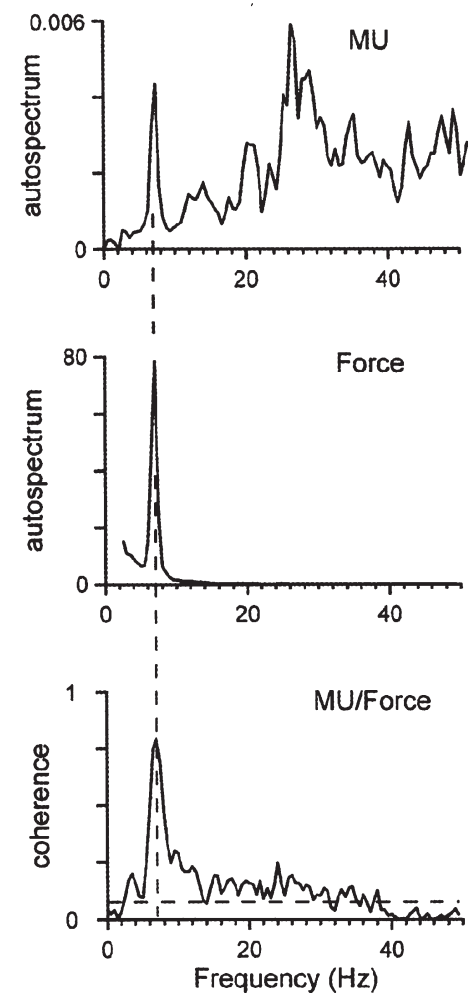

Fig. 3. Quantification of motor units synchronization and firing patterns. Left panels: the motor unit (raw and decomposed traces, intrinsic rate 19 spikes/s) fires triplets and doublets at the tremor frequency $(7 \mathrm{~Hz}$; first dorsal interosseous muscle in a patient). The cross-correlogram shows that doublets/triplets occur mostly around the tremor minima (i.e., "phase-locked to tremor"). Middle panels: The main peak of the first order interspike interval histogram (MU-ISI, uppermost) represents the short ISIs between triplets and doublets (about $40 \mathrm{~ms}$ ). The incidence of triplets (here approximately 80\%) approximately equals the fraction of the third-order intervals (middle histogram, vertical dashed lines) spread over the distribution of the tremor period (lowermost). Right panels: The average synchronization between pairs of motor units in the muscle is quantified by estimating the coherence between autospectra of this motor unit firing and force tremor. Strong correlations occur at the tremor frequency (vertical dashed line). The horizontal dashed line shows the significance limit. MU, motor unit; MVC, maximal voluntary contraction (modified from [44]; Fig. 3, with kind permission).

unit intrinsic rate was twice as high as the tremor frequency, doublets occurred at each tremor cycle (incidence 100\%, motor unit Nr. 2 in Fig. 1). When the motor unit intrinsic rate was less than twice as high as the tremor frequency, doublets interchanged with single discharges, all occurring phase-locked to the tremor cycle (motor unit Nr. 1 in Fig. 1, incidence of doublets $65 \%$ ). Similarly, when the motor unit fired at even higher rates, up to three times the tremor frequency, the spike train consisted of triplets interchanged with doubles (Fig. 3). The quantification of doublet/triplet incidence in relation to the motor unit intrinsic firing rate and the tremor frequency (Fig. 8 in [44] and Fig. 7 in [45]) simply confirmed the qualitative observation that motor units firing at high intrinsic rates are modulated to discharge singlets/doublets/triplets phase-locked to tremor.
Do these repetitive discharges in patients originate from brief augmentations of the motor unit excitatory state? Against this assumption is the experimental finding that the mean short ISI duration was found to be independent from the intrinsic firing rate of the motor unit [45]. Normally, smaller motor units, i.e., those discharging at high rates, are expected to fire at shorter interspike intervals in response to a certain level of impinging excitatory drive than larger units. Most importantly, the longer the ISI duration within doublets/triplets, the greater the difference between the overt (epoch-II) and normal-like (epoch-I) tremor frequency was (Fig. 8D in [45]). This key relationship suggested that doublets/triplets may somehow be linked to a mechanism triggering (lower frequency) parkinsonian out of (higher frequency) physiological tremor. 


\section{INTERACTIONS BETWEEN EXCESSIVE BETA-BAND ACTIVITY AND RHYTHMICAL SPINDLE DISCHARGES CAN MODULATE THE MOTONEURONAL FIRING PATTERN}

As the intrinsic motor unit firing rate does not change across transitions from normal-like epoch-I to overt force and postural tremor epoch-II in patients, the question arises whether physiological tremor can be appropriately modulated to parkinsonian tremor by an input impinging intermittently upon motoneurons. Important clues as to how such modulations may come forth, have been yielded by (i) the invariant ("fixed") mean duration of the short ISI within doublets/triplets across recordings in a given patient and (ii) by the fact that the duration of this interval is positively correlated with the difference between overt and normal-like tremor cycle periods [45]. Indeed, motoneurons synchronized by the afferent Ia bursts and at the same time exposed to (relatively weak) synaptic input in the beta-range, may be expected to discharge spikes separated by approximately $40-50 \mathrm{~Hz}$ just when they are brought near to threshold through the rhythmical spindle afferent activity (Fig. 1). The motor unit force twitch from the resulting paired motoneuron discharges will be then broadened and enlarged as compared to twitches from single discharges (schematic in Fig. 1, see also [52]). Consequently, the delay in the spinal reflex loop will be prolonged (i.e., because of the interposed ISI interval, compare $S 1$ to $S 2$ in the schematic) resulting in oscillations at lower frequency as compared to those during epoch-I normal-like tremor. Also, as the relaxation curve of the enlarged force twitch will be steeper, muscle spindles will, in consequence, discharge more vigorously enhancing thus the synchronizing drive to the motoneuronal pool (Fig. 1). Due to this enhancement, motoneurons will approach discharge threshold more frequently at the instances when both beta-range and spindle synaptic inputs impinge concurrently on their membrane. All spikes in excess of the tremor frequency will be then "bound up" in the formation of doublets/triplets, time-locked to the spinal reflex loop oscillation. Along these lines of argumentation, both the increased synchrony and the enlarged "double" force twitches are thought to be responsible for the larger amplitude of overt parkinsonian tremor. It may be further assumed that proprioceptive afferent inflow via ascending Ia afferent collaterals (e.g., the spinocerebellar tracts, Fig. 1) would drive the cerebello-thalamo-cortical circuit into oscillatory activity such that, in turn, the oscillations within the spinal loop would be reinforced via descending corticomotoneuronal projections. Suppressing beta oscillations (DBS, dopamine) or opening the cerebello-thalamo-cortical circuit (i.e., lesions affecting corticospinal projections, thalamotomy, DBS) remove the trigger and abolish reinforcement respectively. Similarly, opening the spinal reflex loop by immobilizing the trembling extremity has been shown to remove all signs of synchronization of motor unit activity and eliminate or greatly reduce the tremor in patients [53].

There are several limitations of the abovedescribed model. First, details about the specific weights of the interacting inputs and the associated activation of plateau potentials at the level of the motoneuronal membrane are completely missing (cf. [54]). Also, while the finding of "fixed" mean short ISI between doublets/triplets across recordings rules out the possibility that a brief but stable excitation level of the motoneuron is responsible for their generation, it does necessarily prove that these repetitive discharges originate from a rhythmical, beta-range, synaptic input. More robust evidence on their generation mechanism is needed here. Conclusive evidence could be obtained, for example, by correlating the duration of the short ISI with the frequency of betarange oscillations. Interestingly, the spectral profile of beta-range oscillations in STN, though variable among patients, has been shown to remain stationary over longer time periods in a given patient such as to characterize a 'signature' rhythm [55]. Thus, correlations with the short 1SI, likewise invariant for a given patient, can be possibly estimated by recording both intramuscular EMG and basal ganglia local field potentials simultaneously in the same patient.

The main evidence that parkinsonian tremor cannot be caused by peripheral mechanisms stems from early studies stating that extensive posterior root section or novocain anaesthesia of tremulous muscles do not abolish the tremor in patients $[56,57]$. However, detailed recordings under controlled conditions were at that time, almost a century ago, not obtainable. The possible involvement of the monosynaptic stretch reflex loop in tremor generation and motor unit synchronization could be re-examined non-invasively through short-term ischemia of the tremulous limb. There is evidence that tourniquetischemia over 15 min blocks selectively the feedback from large Ia afferents conveying signals from the muscle spindles [58-60]. 
Additional assumptions are, however, needed to explain triggering of resting tremor by the proposed putative mechanism because motor units may not, theoretically, discharge at rest and be the substrate for the interactions of the spinal reflex loop/ beta-band activities. Patients experience, nevertheless, great difficulty when they are asked to relax. It has been shown under controlled conditions favouring muscle relaxation that low threshold motor units in hand muscles are discharging in patients even for $15 \mathrm{~min}$ after the command to relax [61]. Still, further studies are needed to clarify additional mechanisms of resting tremor generation.

\section{CONCLUSION}

The search for a mechanism acting as an interface between the dopamine depleted basal ganglia and cerebello-thalamo-cortical oscillating circuits, both involved in parkinsonian tremor generation, has been so far inconclusive. Several reports have, however, pointed to the relevance of interactions of central oscillations with peripheral/spinal mechanisms. Recently, the quantification of motor unit synchrony and firing patterns in patients suggested that the triggering mechanism (the "node", [62]) may be at the level of spinal motoneurons. According to the proposed mechanism, exaggerated beta-range oscillations impinge intermittently onto the membrane of motoneurons which, thereupon, fire doublets/triplets when concurrent excitatory input from Ia afferents brings them periodically near to threshold. Enlarged force twitches evoked from doublets/triplets will, in turn, augment grouping of motor units due to enhanced muscle spindle activation. At the same time delays in the signal transmission loop are prolonged because of the extended twitch contraction time. These modifications are, in turn, transmitted to supraspinal structures and the oscillations at the spinal/peripheral level are maintained/amplified by larger transcortical loops. Further evidence is, however, needed to prove the validity of the proposed interactions.

\section{ACKNOWLEDGMENTS}

Most of the presented views on parkinsonian tremor generation emerged through numerous insightful discussions with the late Professor C. Christakos and PhD student Miss O. Agapaki.

\section{CONFLICT OF INTEREST}

The author has no conflict of interest to report

\section{REFERENCES}

[1] Albin RL, Young AB, Penney JB (1989) The functional anatomy of basal ganglis disorders. Trends Neurosci 12, 366-375.

[2] Louis ED, Levy G, Côte LJ, Mejia H, Fahn S, Marder K (2001) Clinical correlates of action tremor in Parkinson disease. Arch Neurol 58, 1630-1634.

[3] Zetusky WJ, Jancovic J, Pirozzolo Fj (1985) The heterogeneity of Parkinson's disease: Clinical and prognostic implications. Neurology 35, 522-526.

[4] Fishman PS (2008) Paradoxical aspects of parkinsonian tremor. Mov Disord 23, 168-173.

[5] Obeso JA, Marin C, Rodriguez-Oroz C, Blesa J, BenitezTemino B, Mena-Segovia J, Rodriguez M, Olanov CW (2008) The basal ganglia in Parkinson's disease: Current concepts and unexplained observations. Ann Neurol 64(Suppl 2), S30-S46.

[6] Zirh TA, Lenz FA, Reich SG, Dougherty PM (1998) Patterns of bursting occurring in thalamic cells during parkinsonian tremor. Neuroscience 83, 107-121.

[7] Tasker RR (1990) Thalamotomy. Neurosurg Clin N Am 1, 841-864.

[8] Fox MW, Ahlskog JE, Kelly PJ (1991) Stereotactic ventrolatelalis thalamotomy for medically refractory tremor in post-levodopa era Parkinson's disease patients. J Neurosurg 75, 723-730.

[9] Benabid AL, Pollak P, Gervason C, Hoffmann D, D.M. Gao DM, Hommel M, Perret JE, de Rougemont J (1991) Longterm suppression of tremor by chronic stimulation of the ventral intermediate thalamic nucleus. Lancet 337, 403-406.

[10] Lenz FA, Normand SL, Kwan HC, Andrews D, Rowland LH, Jones MW, Seike M, Lin YC, Tasker RR, Dostrovsky JO (1995) Statistical prediction of the optimal site for thalamotomy in parkinsonian tremor. Mov Disord 10, 318-328.

[11] Hutchison WD, Lozano AM, Tasker RR, Lang AE, Dostrovsky JO (1997) Identification and characterization of neurons with tremor-frequency activity in human globus pallidus. Exp Brain Res 113, 557-563.

[12] Hurtado JM, Rubchinsky LL, Sigvardt KA, Wheelock VL, Pappas CT (2004) Temporal evolution of oscillations and synchrony in GPi/muscle pairs in Parkinson's disease. $J$ Neurophysiol 93, 1569-1584.

[13] Rivlin-Etzion M, Marmor O, Heimer G, Raz A, Nini A, Bergman H (2006) Basal ganglia oscillations and pathophysiology of movement disorders. Curr Opin Neurobiol 16, 629-637.

[14] Lozano AM, Lang AE, Hutchison WD (1998) Pallidotomy for tremor. Mov Disord 13(Suppl 3), 107-110.

[15] Helmich RC, Janssen MJ, Oyen WJ, Bloem BR, Toni I (2011) Pallidal dysfunction drives a cerebellothalamic circuit into Parkinson tremor. Ann Neurol 69, 269-281.

[16] Helmich RC, Hallett M, Deuschl G, Toni I, Bloem B (2012) Cerebral causes and consequences of parkinsonian resting tremor: A tale of two circuits? Brain 135, 3206-3226.

[17] Brown P, Williams D (2005) Basal ganglia local field potential activity: Character and functional significance in the human, Clin Neurophysiol 116, 2510-2519. 
[18] Hammond C, Bergman H, Brown P (2007) Pathological synchronization in Parkinson's disease: Networks, models and treatments. Trends Neurosci 30, 357-364.

[19] Bronte-Stewart H, Barberini C, Koop MM, Hill BC, Henderson JM, B. Wingeier B (2009) The STN beta-band profile in Parkinson's disease is stationary and shows prolonged attenuation after deep brain stimulation. Exp Neurol 215, 20-28.

[20] Kühn AA, Kupsch A, Schneider GH, Brown P (2006) Reduction in subthalamic $8-35 \mathrm{~Hz}$ oscillatory activity correlates with clinical improvement in Parkinson's disease. Eur J Neurosci 23, 1956-1960.

[21] Bergman H, Wichmann T, Karmon B, DeLong MR (1994) The primate subthalamic nucleus. II. Neuronal activity in the MPTP model of parkinsonism. J Neurophysiol 72, 507-520.

[22] Liu X, Ford-Dunn HL, Hayward GN, Nandi D, Miall RC, Aziz TZ, Stein JF (2002) The oscillatory activity in the parkinsonian subthalamic nucleus investigated using the macro-electrodes for deep brain stimulation. Clin Neurophysiol 113, 1667-1672.

[23] Amtage F, Henschel K, Schelter B, Vesper J, Timmer J, Lücking CH, Hellwig B (2008) Tremor-correlated neuronal activity in the subthalamic nucleus of Parkinsonian patients, Neurosci Lett 442, 195-199.

[24] Florin E, Himmel M, Reck C, Maarouf M, Schnitzler A, Sturm V, Fink GR, Timmermann L (2012) Subtype-specific statistical causalities in parkinsonian tremor. Neuroscience 210, 353-362.

[25] Hirschmann J, Abbasi O, Storzer L, Butz M, Hartmann CJ, Wojteck L, Schnitzler A (2019) Longitudinal recordings reveal transient increase of Alpha/Low-Beta power in the subthalamic nucleus associated with the onset of parkinsonian rest tremor. Front Neurol 10, 145.

[26] Rack PMH, Ross HF (1986) The role of reflexes in the resting tremor of Parkinson's disease. Brain 109, 115-141.

[27] Teravainen H, Evarts E, Calne D (1979) Effects of kinesthetic inputs on parkinsonian tremor. Adv Neurol 24, 161-173.

[28] Lee RG, Stein RB (1981) Resetting of tremor by mechanical perturbations: A comparison of essential tremor and parkinsonian tremor. Ann Neurol 10, 523-531.

[29] Britton TC, Thompson PD, Day BL, Rothwell JC, Findley LJ, Marsden CD (1992) "Resetting" of postural tremors at the wrist with mechanical stretches in Parkinson's disease essential tremor and normal subjects mimicking tremor. Ann Neurol 31, 507-514.

[30] Hufschmidt HJ (1963) Proprioceptive origin of parkinsonian tremor. Nature 200, 367-368.

[31] Britton TC, Thompson PD, Day BL, Rothwell Jc, Findley LJ, Marsden CD (1993) Modulation of postual tremors at the wrist by supramaximal electrical median nerve shocks in essential tremor, Parkinson's disease and normal subjects mimicking tremor. J Neurol Neurosurg Psychiatr 56, 10851089.

[32] Britton TC, Thompson PD, Day BL, Rothwell JC, Findley LJ, Marsden CD (1993) Modulation of postual tremors by magnetic stimulation of the motor cortex in patients with Parkinson's disease or essential tremor, and in normal subjects mimicking tremor. Ann Neurol 33, 473-479.

[33] Pascual-Leone A, Valls-Solé J, Toro C, Wassermann EM, Hallett M (1994) Resetting of essential tremor and postural tremor in Parkinson's disease with transcranial magnetic stimulation. Muscle Nerve 17, 800-807.

[34] Ni Z, Pinto AD, Lang AE, Chen R (2010) Involvement of the cerebellothalamocortical pathway in Parkinson disease. Ann Neurol 68, 816-824.
[35] Halliday AM, Redfearn JWT (1956) An analysis of the frequencies of finger tremor in healthy subjects. J Physiol 134, 601-611.

[36] Lippold OCJ (1970) Oscillation in the stretch reflex and the origin of the rhythmical, $8-12 \mathrm{c} / \mathrm{s} \mathrm{component} \mathrm{of} \mathrm{physiolog-}$ ical tremor. J Physiol 206, 359-382.

[37] Hagbarth KE, Young RR (1979) Participation of the stretch reflex in human physiological tremor. Brain 102, 509-526.

[38] Negro F, Orizio C (2017) Robust estimation of average twitch contraction forces of populations of motor units in humans. J Electromyogr Kinesiol 37, 132-140.

[39] Sears TA, Stagg D (1976) Short-term synchronization of intercostal motoneurone activity. J Physiol 263, 357-381.

[40] Dengler R, Wolf W, Schubert M, Struppler A (1986) Discharge pattern of single motor units in Parkinson's disease. Neurology 36, 1061-1066.

[41] Baker JR, Davey NJ, Ellaway PH, Friedland CL (1992) Short-term synchrony of motor unit discharge during weak isometric contraction in Parkinson's disease. Brain 115, 137-154.

[42] Das Gupta A (1963) Paired response of motor units during voluntary contraction in Parkinsonism. J Neurol Neurosurg Psychiatr 26, 265-268.

[43] Dietz V, Hillesheimer W, Freund HJ (1974) Correlation between tremor, voluntary contraction and firing pattern of motor units in Parkinson's disease. J Neurol Neurosurg Psychiatr 37, 927-937.

[44] Christakos CN, Erimaki S, Anagnostou E, Anastasopoulos D (2009) Tremor-related motor unit firing in Parkinson's disease: Implications for tremor genesis. J Physiol 587, 4811-4827.

[45] Agapaki O, Christakos CN, Anastasopoulos D (2018) Characteristics of rest and postural tremors in Parkinson's disease: An analysis of motor unit firing synchrony and patterns. Front Hum Neurosci 12, 179.

[46] Christakos CN (1997) On the detection and measurement of synchrony in neural populations by coherence analysis, J Neurophysiol 78, 3453-3459.

[47] Farina D, Negro F, Jiang N (2013) Identification of common synaptic inputs to motor neurons from the rectified electromyogram. J Physiol 591, 2403-2418.

[48] Perkel DH, Gerstein GI, Moore GP (1967) Neuronal spike trains and stochastic point processes. I. The single spike train. Biophys J 7, 391-418.

[49] Christakos CN, Erimaki S, Anagnostou E, Anastasopoulos D (2010) Analysis of motor unit firing patterns and synchrony contribute to our understanding of tremor mechanisms in Parkinson's disease. Physiol News 79, 28-30.

[50] Heckman CJ, Mottram C, Quinlan K, Theiss R, Schuster J (2009) Motoneuron excitability: The importance of neuromodulary inputs. Clin Neurophysiol 120, 2040-2054.

[51] Kudina LP, Andreeva RE (2019) Repetitive doublet firing in human motoneurons: Evidence for interaction between common synaptic drive and plateau potential in natural motor control. J Neurophysiol 122, 424-434.

[52] Thomas CK, Johamsson RS, Bigland-Ritchie B (1999) Pattern of pulses that maximize force output from single human thenar motor units. J Neurophysiol 82, 3188-3195.

[53] Burne JA (1987) Reflex origin of parkinsonian tremor. Exp Neurol 97, 327-339.

[54] Bennett D, Hultborn H, Fedirchuk B, Gorassini M (1998) Synaptic activation of plateaus in hindlimb motoneurons of decerebrate cats. J Neurophysiol 80, 2023-2037.

[55] Bronte-Stewart H, Barberini C, Miller Koop M, Hill B, Henderson J, Wingeier B (2008) The STN beta-band profile 
in Parkinson's disease is stationary and shows prolonged attenuation after deep brain stimulation. Exp Neurol 215, 20-28.

[56] Walshe FMR (1924) Observations on the nature of the muscular rigidity of paralysis agitans, and on its relationship to tremor. Brain 47, 159-177.

[57] Pollock LJ, Davis L (1930) Muscle tone in parkinsonian states. Arch Neurol Psychiatry 23, 303-319.

[58] Fellows SJ, Doemges F, Topper R, Thilmann AF, Noth J (1993) Changes in the short- and long-latency stretch reflex components of the triceps surae muscle during ischaemia in man. J Physiol 472, 737-748.
[59] Christakos CN, Papadimitriou N, Erimaki S (2005) Parallel neuronal mechanisms underlying physiological force tremor in steady muscle contractions of humans. J Neurophysiol 95, 53-66.

[60] Hofmeijer J, Franssen H, van Schelven L, van Putten M (2013) Why are sensory axons more vulnerable for ischemia than motor axons? PLoS One 8, e67113.

[61] Cantello R, Gianelli M, Civardi C, Mutani R (1995) Parkinson's disease rigidity: EMG in a small hand muscle at "rest". Electroenceph Clin Neurophysiol 97, 215-222.

[62] Hallett M (2014) Tremor: Pathophysiology. Parkinsonism Relat Disord 20 (Suppl 1), 118-122. 